\title{
Agentes patógenos en el síndrome diarreico agudo no enterocólico
}

\author{
Dr. Juan Gutiérrez B.; Dr. Humberto Soriano B.; Dr. Miguel O'Ryan G.; \\ Dr. Pablo Avendaño L.; Dra. Karis D'Ottone M.; \\ Dr. Humberto Soriano P.
}

\section{Potentially pathogenic agents in non enterocolic diarrhea}

\begin{abstract}
Along a one year period 112 infants admitted with non enterocolic acute diarrhea were studied for isolation of potentially ethiologic agents, namely enteropathogenic bacterin (Salmonella, Shigella, Campylobacter, classic enteropathogenic, enteroinvasive and enterotoxigenic Escherichia coli), Rotovinus (viral RNA electrophoresis) and enteroparasites (Telemann and PAFS). The most frequently identified pathogen was rotavirus (57.8\%), followed by thermo labile toxin producing Escherichia coli $(19.7 \%)$. The frecuency of classic enteropathogenic Escherichio coli was $13.9 \%$, that of thermo stable toxin producing Escherichio coli $5.7 \%$, Shigella $4.1 \%$, Campylobacter $3.3 \%$ and Salmonella 1.6\%. Bacteriae were isolated from $40.2 \%$, of patients, predominantly in summer. Enteroparasites were detected in $13.1 \%$ of the cases, Entamoeba histolytica being the most frequent. [n $32.8 \%$ of the cases more than one pathogen was isolated.
\end{abstract}

(Key words: non enterocolic diarriea, microbiology.)

La etiología del sindrome diarreico agudo (SDA) del niño pequeño ha constituido siempre un punto de particular interés para el pediatra. Los avances de los últimos años han permitido la identificación de un número creciente de agentes patógenos que han sido implicados en la génesis del SDA.

El objetivo de nuestro estudio fue la identificación de los posibles agentes causantes del SDA no disentérico del niño que se hospitaliza por deshidratación, usando una completa batería de exámenes, la cual abarca la mayor parte de los gérmenes que hasta ahora se han menciona. do como los agentes etiológicos más frecuentes (tabla 1). Quedan excluidos el Cryptosporidium, la Escherichia coli enteroadhesiva y la Yersinia enterocolitica, los primeros por ser patógenos relativamente nuevos, cuya real importancia no ha sido claramente establecida hasta el momento, y la última porque en la experiencia nacional $^{1,2}$, y en la personal, no ha sido encontrada sino excepcionalmente en ninos con SDA.

- Servicio de Pediatría. Hospital Dr. Sótero del Rio.

- Servicio de Pediatría. Hospital Paula Jaraquemada.

- Laboratorio de Referencia de Enterobacterias del Instituto de Salud Pública.

- Departamento de Pediatría. Escuela de Medicina de la Pontificia Universidad Católica de Chile.

\section{MATERLALES Y METODOS}

Se estudió un grupo de niños de 4 a 36 meses de edad que ingresaton a los servicios de pediatr f́a de los hospitales Paula Jaraquemada y Dr. Sótero del Río, por cuadros diarreicos sin sangre, de menos de $72 \mathrm{~h}$ de evolución, que no habían recibido antibióticos y no presentaban evidercias de enfermedad crónica o infección bacteriana concomitante en otro territorio. Se excluyó también a los desnutridos severos y a los

Tabla 1

Agentes etiológicos más frecuentes en el sindrome diarreico agudo del lactante

\footnotetext{
Escherichia coli

Serotipos enteropatógenos clásicos

Enterotoxigénica toxina termolábil.

Enterotoxigénica toxina termoestable.

Enteroinvasora (Shigella like).

Enteroadhesiva.

Shigella

Salmonella

Campylobacter jejuni

Yersinta enterocolitica
}

\section{Rotovirus}

Giardia lamblia

Entamoeba histolytica

Cryptosporidium 
que presentaton "shock" al ingreso. El estudio se extendió desde el 15 de diciembre de 1985 al 15 de diciembre de 1986.

Al ingreso se tomó una muestra de deposición para estudio bacteriológico (Escherichia coli, Salmonella, Shigella. Campylobacter), que se realizó en el laboratorio de microbiologia de la Pontificia Universidad Católica de Chile de acuerdo a técnicas estándar. Desde allí se enviaron muestras al laboratorio de referencia de enterobacterias del Instituto de Salud Pública de Chile, donde se efectuó el estudio para la detección de las cepas enteropatógenas clásicas (serotipificacjón), enteroinvasoras (prueba de Sereny) ${ }^{3}$ y determinación de enterotoxinas termolábil (ELISA GM I) ${ }^{4}$ y termoestable (método de Dean) ${ }^{5}$ de Escherichia coll.

La búsqueda de Rotavins se efectuó mediante muestras tomadas el primer, tercer y quinto días, analizadas por electroforesis del ARN viral en gel de poliacrilamida (técnica de Espejo) ${ }^{6}$, procedimiento realizado en laboratorios del Hospital Paula Jaraquemada. Se consideró que el paciente tenía una diarrea por Rotavirus sólo si las muestras del primer o tercer día - ambos estaban positivas, a fin de descartar las posi. bles infecciones intrahospitalarias.

Se recolectaron deposiciones durante los tres primeros días para estudio coproparasitológico (técnicas de Telemann y PAFS modificado) efectuado en el laboratorio de parasitologia de la Pontificia Universidad Católica de Chile.

El análisis estadístico de los datos se realizó con la técnica de chi cuadrado.

\section{RESULTADOS}

Se reclutaron 139 pacientes, de los cuales 17 fueron posteriormente retirados por alguno de los criterios de exclusión: en 7 de ellos apareció evidencia de infección bacteriana en otro territorio; tres presentaron deposiciones con sangre (en uno de ellos apareció Shigella en el copro. cultivo). En otros 3 pacientes se hizo evidente una enfermedad crónica o requirieron traslado a otro servicio. Dos casos fueron retirados por razones no médicas (decisión de los padres). Se incluyeron en el análisis 122 pacientes, cuyas características se describen en la tabla 2.

Se detectó algún patógeno potencialmente causante de diarrea en $80,3 \%$ de los casos (tabla 3). Los microorganismos encontrados con más frecuencia fueron: Rotavirus $(57,8 \%)$ y Escherichia coli enterotoxina termolábil (LT) $(19,7 \%)$, seguidos por las cepas enteropatógenas clásicas de $E$. coll $(13,9 \%)$ (predominando los serotipos 0111 y 086). Hubo $5,7 \%$ de hallazgo de $E$. coli enterotoxina termoestable (ST). La frecuencia de Campylobacter fue de $3,3 \%$, y la del total de bacterias potencialmente
Tabla 2

Caracter ísticas generales de la población

\begin{tabular}{|c|c|c|}
\hline & $\begin{array}{l}\text { Número de } \\
\text { pacientes }\end{array}$ & $\begin{array}{c}\text { Porcentaje } \\
\text { del total } \\
\%\end{array}$ \\
\hline \multicolumn{3}{|l|}{ PROCEDENCIA } \\
\hline Hosp. Dr. Sátero del Río & 62 & 50,8 \\
\hline Hosp. Paula Jaraquemada & 60 & 49,2 \\
\hline \multicolumn{3}{|l|}{ SEXO } \\
\hline Masculino & 74 & 60.7 \\
\hline Fernenino & 48 & 39,3 \\
\hline \multicolumn{3}{|l|}{$\mathrm{EDAD}$} \\
\hline Promedio & 9,8 meses & \\
\hline Desviación estándar & 6.0 meses & \\
\hline Rango & $4,0-28,0$ meses & \\
\hline \multicolumn{3}{|l|}{$\begin{array}{l}\text { EST ADO NUTRITIVO } \\
\text { (Según tablas de peso/edad } \\
\text { de la NCHS) }\end{array}$} \\
\hline p $5-25$ & $38.5 \%$ & \\
\hline p $25-75$ & $58,2 \%$ & \\
\hline p mayor que 75 & $3,3 \%$ & \\
\hline
\end{tabular}

invasoras de la pared fue de $9,0 \%$. No se encontró ninguna $E$. coli enteroinvasora. Los entero. parásitos se detectaron con una frecuencia de 13,1\% (11 casos de Entamoeba histolytica, 3 casos de Giardia lamblia y 2 casos de infec. ción doble) (tabla 3).

No se encontró diferencia significativa en la frecuencia de hallazgo de patógenos ni en el tipo de éstos al comparar por sexos, edades o centros hospitalarios.

En alto porcentaje de los casos se encontro asociación de dos o más gérmenes en un mismo paciente $(32,8 \%)$, especialmente en los meses de calor $(41,9 \%$ en el período septiembre-febrero; $18,8 \%$ en el período marzo-agosto, diferencia significativa $(p<0,05)$. La asociación más frecuente fue la de Rotavirus con una bacteria. No se detectaron diferencias significativas al analizar este aspecto por sexos, edades o centros hospitalarios.

No se encontró diferencia estadísticamente significativa al comparar la frecuencia de detección de patógenos durante los meses más fríos con la de los meses más cáliđos, aunque el número de diarreas durante los primeros fue menor (48 casos en el período marzo-agosto, con 
Tabla 3

Fiecuencia de hallazgo de enteropatógenos en el síndrome diarteico agudo no enterocólico. diferencjado según el per ́́odo del año

\begin{tabular}{|c|c|c|c|c|c|c|}
\hline \multirow[b]{3}{*}{ Número de pacientes } & \multicolumn{2}{|c|}{$\begin{array}{l}\text { Septiembre- } \\
\text { febrero }\end{array}$} & \multicolumn{2}{|c|}{$\begin{array}{l}\text { Marzo- } \\
\text { agosto }\end{array}$} & \multirow{3}{*}{$\begin{array}{r}\text { Total } \\
122\end{array}$} & \multirow{3}{*}{$\begin{array}{l}\% \text { del total } \\
100,0\end{array}$} \\
\hline & No & $\%$ & $\mathrm{~N}^{\circ}$ & $\%$ & & \\
\hline & \multicolumn{2}{|l|}{74} & \multicolumn{2}{|l|}{48} & & \\
\hline Con patógeno & 62 & $(83,8)$ & 36 & $(75,0)$ & 98 & 80,3 \\
\hline Sin patógeno & 12 & $(16,2)$ & 12 & $(25,0)$ & 24 & 19,7 \\
\hline Con dos o más patógenos & 31 & $(41,9)$ & 9 & $(18,8)$ & 40 & 32,8 \\
\hline Rotavinus (*) & 42 & $(57,5)$ & 28 & $(58,3)$ & 70 & 57,8 \\
\hline Bacterias & 35 & $(47,3)$ & 14 & $(29,2)$ & 49 & 40,2 \\
\hline - E. coli serotipos ctásicos (**) & 12 & $(16,4)$ & $s$ & $(10,4)$ & 17 & 13,9 \\
\hline - E. coli LT & 18 & $(24,3)$ & 6 & $(12,5)$ & 24 & 19,7 \\
\hline$-E$ coll ST & 6 & $(8,1)$ & 1 & $(2,1)$ & 7 & 5,7 \\
\hline - Shigella & 5 & $(6,8)$ & 0 & $(-)$ & 5 & 4,1 \\
\hline S. flexneri & & & & & 3 & \\
\hline S. sonnei & & & & & 2 & \\
\hline - Campylobacter & 1 & $(1,4)$ & 3 & $(6,2)$ & 4 & 3,3 \\
\hline - Salmonella & 1 & $(1,4)$ & 1 & $(2,1)$ & 2 & 1,6 \\
\hline S. typhi & & & & & 1 & \\
\hline S. paratyphi & & & & & 1 & \\
\hline Enteroparásitos & 13 & $(17,6)$ & 3 & $(6,2)$ & 16 & 13,1 \\
\hline - E. histolytica & & & & & 11 & 8,8 \\
\hline - Giardia lamblia & & & & & 3 & 2,5 \\
\hline- E. Histolytica + G. tamblio & & & & & 2 & 1,6 \\
\hline
\end{tabular}

(*) En un caso no se completó el estudio de Rotayirus.

(**) Un caso đe infección doble (serotipos $086+055$ ).

Abreviaturas:

$\mathrm{LT}=$ Enterotoxina termolábil.

ST = Enterotoxina termoestable.

$75 \%$ de detección de gérmenes; 74 casos en el período septiembre-febrero, con $83,8 \%$ de detección de gérmenes).

El patógeno detectado con más frecuencia en los meses invernales fue Rotavirus $(58,3 \%)$ (tabla 3), que mantiene una frecuencia relativamente pareja a lo largo de todo el año. En los Ineses de verano $\mathrm{va}$ aumentando la frecuencia de las bacterias ( $p<0,0005$ ). Respecto de los enteroparásitos, sólo 3 de los 16 casos encontrados correspondieron al periodo marzo-agosto.

\section{DISCUSION}

Este estudio, a diferencia de otros publica$\operatorname{dos}^{7-22}$, abarca en forma concomitante un ann. plio rango de agentes patógenos.

La alta frecuencia de detección de gérmenes en este estudio demuestra que, si se usan los métodos apropiados disponibles, podemos llegar en nuestra población al diagnóstico etiológico probable en alrededor de $80 \%$ de los SDA no disentéricos de niños pequeños que se hospitalizan 
por deshidratación, frecuencia mayor que lá re. portada en otros estudios de búsqueda etiológica menos amplia y diferentes criterios en la selección de los pacientes ${ }^{12,17,22,23}$

La frecuencia de Rotavirus es alta en relación con la encontrada en otros estudios, 10, 12, 16-21. En dos trabajos previos realizados con lactantes de la misma área geografica la frecuencia de Rotavinus fue menor ${ }^{17,18}$. Esto puede explicarse por el criterio de selección usado en nuestra investigación, que consideró sólo las diarreas agudas no disentéricas de menos de $72 \mathrm{~h}$ de evolución con deshidratación y por el perfeccionamiento de las técnicas de diagnóstico. En trabajos previos no se han descrito casos de portación asintomática de Rotavirus" ${ }^{8,}$, excepto en uno ${ }^{9}$. Esto, sumado al hecho de que en $80 \%$ de los casos en que se encontró el virus éstos desaparecieron de las muestras al quinto día, sugiere fuertemente que se trataba de infecciones agudas.

El alto número de casos de asociación de patógenos podría reflejar mayor exposicjón ambiental de algunos pacientes, condicionada, probablemente, por factores socioeconómicos. Sin embargo, nuestra población no permitió establecer diferencias claras en este aspecto, dada la pobreza relativamente homogénea de los grupos dependientes de los servicios de salud estatales. No detectamos diferencias al analizarlo por sexo y edad. En invierno la frecuencia de estas infecciones múltiples es significativamente más baja ( $\mathrm{p}<$ que 0,05 ), presumiblemente por descenso en la exposición a bacterias patógenas, situación concordante con otras publicaciones ${ }^{1,12}$.

E1 segundo germen en frecuencia fue Escheri. chia coli LT, superando largamente a la $E$. coli ST y a las cepas enteropatógenas clásicas (tabla 3). Este es un hecho nuevo, difícil de comparar con estudios previos, pues las $E$. coli enterotoxigénicas han sido relativamente poco estudiadas en nuestro media. Hay una publicación en que la importancia de este patógeno era bastante menor $^{25}$. La $E$. coli enteropatógena clásica, tradicionalmente reconocida como el agente etiológico más importante en el SDA infantil $^{11} 2,7,8,12,14,15,17,20,25$, alcanzó una fre. cuencia de sólo $13,9 \%$, predominando los serotipos 0111 y 086. Recordemos, además, que se han descrito muchos casos de infecioiones asintomáticas con estas bacterias ${ }^{10,15,20,23}$, La $E$. coli ST se detectó con una frecuencia de $5,7 \%$, comparable con la señalada en otro trabajo $^{25}$.
Respecto de las bacterias potencialmente invasoras de la pared (Campylobacter, Shigella, Satmonella), como era de esperar, dada la exclusión de los SDA enterocólicos, alcanzaron niveles bajos, aunque no despreciables, de detección $(9,0 \%$ en total). No se detectó ninguna $E$. coli enteroinvasora. El Campylobacter, más frecuente en las diarreas ambulatorias, se detectó, en general, menos que en estudios pre$\operatorname{vios}^{1,2,8,13,15,17,26}$. Llama la atención el hallazgo de 4,1\% de Shigella en pacientes sin enterocolitis, de manera que la ausencia de sangre en las deposiciones no debe infundir una falsa seguridad respecto de la no existencia de este patógeno.

La frecuencia de detección de enteroparásitos fue similar o menor que en investigaciones anteriores $^{22,23,27-31}$, pero, a diferencia de la mayoria de ellas, en que el parásito predominante era Giardia lamblia, en ésta pređominó claramente la Entamoeba histolytica. Cabe destacar que la frecuencia de este último parásito probablemente está incluso subestimada, pues en un estudio reciente ${ }^{11}$, en que también fue el parásito más frecuente en los lactantes, se demostró la importancia que tiene el examen de muestras frescas para detección de trofozoí. tos de ameba, procedimiento no efectuado en este trabajo. No se encontraron otros enteroparásitos aparte de los mencionados. En dos informes previos sobre lactantes con SDA del mismo sector geográfico no se encontró ningún parásito ${ }^{12,17}$.

Como conclusión, podriamos establecer que la etiología del SDA no disentérico, de menos de $72 \mathrm{~h}$ de evolución, en lactantes que se hospitalizan por deshidratación, es muy variada, y que esta etiología es posible de determinar en $80 \%$ o más de estos pacientes. El agente causal, en por lo menos $50 \%$ de los casos en que se detecta, sería susceptible de tratar con antibacterianos no absorbibles o con antiparasitarios específicos, como coadyuvante al tratamiento de rehidratación; luego, debiera ser una práctica establecida el buscar la etiología de todo SDA intenso que amenace con deshidratación toda vez que ello sea posible.

\section{RESUMEN}

Se estudiaron 112 lactantes hospitalizados por síndrome diarteico agudo no enterocólico en el periodo de un aflo, a fin de detectar los po- 
sibles agentes etiológicos (Salmonella, Shigella, Campylobacter, Escherichia coli enteropatógeno clásico, enterotoxigénico y enteroinvasor), Rotavirus (electroforesis del ARN viral) y enteropa. rásitos (Telemann y PAFS).

Rotavinus fue el patógeno detectado con más frecuencia $(57,8 \%)$, manteniérdose constante durante todo el año. El segundo germen en inn. portancia fue Escherichia coli enterotoxigénico toxina termolábil $(19,7 \%)$. La frecuencia de $E$. coli enteropatógeno clásico fue de 13,9 por ciento, $E$. coll enterotoxigénico toxina termoestable 5,7\%, Shigella 4,1\%, Campylobacter 3,3\%, Salmonella 1,6\%. La frecuencia del total de bacterias fue de $40,2 \%$, siendo mayor en verano. Los enteroparásitos se encontraron en 13,1\% de los casos, predominando la Entamoeba histolytica. En $32,8 \%$ de los casos se detectó más de un patógeno.

Se discuten los resultados, comparándolos con reportes previos.

\section{AGRADECIMIENTOS}

Deseamos expresar nuestros agradecimientos a la señorita Luz Zapata, tecnólogo médico, por su valiosa labor en la tipifícación de Escherichia coli, y a las enfermeras y personal auxiliar de los servicios de pediatría de los hospitales Paula Jarayuemada y Dr. Sótero del Río, que colaboraron en la realización de este estudio.

\section{REFERENCIAS}

1. Garcia J., Maldonado A., Volenzuela $M$. et al. Aislamiento de Clostridium difficile, Campylobacter fetus sp jejuni y Yersinia enterocolitica desde diarrea aguda. Bol ISP Chile 1982;23; 10-15.

2. Garcia $J$, Maldonado $A$,, Lagos $R$. et al. Primer aislamiento en Chile de Campylobacter fetus sp jejuni y Yersiniz enterocolitica desde deposición de niños menores de 2 años de edad con diarrea aguda. Bol ISP Chile 1981; 22: 23-26.

3. Sereny B.: Experimental Shigella keratoconjuntivitis, Acta Microbiol Acad Scient Hung 1955; 2: 293-296.

4. Sieneyholm A., Humgren J.: ldentification of Escherichia coli heat labile enterotoxin by means of a ganglioside immunoabsorbent assiy (GM I ELISA) procedure, Curr Microbiol 1978; 1: 19-23.

5. Deun A., Chuan Ching Y., William R., Harden L.: Test for Escherichia coli enterotoxin using infant mice: application in a study of diarrhea in children in Honolulu. J Infect Dis $1972 ; 125: 407-411$.

6. Espejo R., Romero P., Calderón E., González $Y_{\text {.: }}$ Diagnóstico de Rotavirus por electroforesis del RNA viral, Bol Med Hosp Infant Mex 1978; 35 : 323-331.

7. Henriquez M., Venegas G., Soto G., Rudolff $T$, Salos J.: Etiología bacteriana de la diarrea aguda del lactante en otono e invierno. Rev Chil Pediatr $1985 ; 56: 450-453$.

8. Prado V., Braun $\$$, Bosch P., Bercovich $M$., Reyes $L$., Sawada M.: Análisis de Escherichia coli enteropatógeno clásico como causa endémica de diarrea aguda en niños chilenos. Rev Chil Pediate 1984; 55: 171.175 .

9. Aroya M., Spencè E., Brunser O, Espinoza J., Sandino A.: Estudio comparativo de dos métodos en el diagnóstico de Rotavirus en lactantes con diarres aguda $y$ asintomáticos. Rev Chil Pediatr 1985 ; 56 : 441444 .

10. Calderón A., Macaya J., Avendaño L., Prenzel I., Ojedo J, Duarte E.: Diarrea aguda por Rotavirus: algunos aspectos clínicos. Rev Chil Pediatr 1980; $51 ; 113-116$.

11. Muñoz P., Saclzer E.,Schilling E., Atios A., Enriquez $N$., Escobar E.: Importancia de las enteroparasitosis como causa del sínd rome diarreico agudo del lactante. Rev Chj Pediatr 1980; 51;407-410.

12. Zocarias J., Spencer E., Prado V., et al. Recuperación fecal de Rotavirus y otros enteropatógenos en lactantes menores de un año con diarrea aguda. Rev Chil Pediatr 1982; 53 : 111-116.

13. Duffau G., Lagos R., Gorcio J.. Maldonado A.: Campylobacter fetus sp jejuni en lactantes con síndrome diarreico agudo. Rev Chil Pediatr 1982;53; 205-208.

14. Prado V., Mimica I., Eduardo M.: Etiología bacteriana de la djarrea aguda del lactante. Aportes de la inmunofluorescencia. Estudios de sensibilidad in vitro de los bacterjos patógenos aislados. Rev Chil Pediatr $1974 ; 45: 135-142$.

15. Prado $V_{\text {.: }}$ Epidemiología de la enteritis por Campylobacter en niños. Rev Med Chile 1984; 112: 1153.

16. Avendan̄o L., Calderón A., Vangas $S_{\text {.: }}$ Rotavirus en diarrea aguda. Estudio comparativo en lactantes hospitalizados $y$ ambulatorios. Rev Med Chile 1981; 109: 303-305.

17. Figueroa G., Aroya M., Ibúnez S. Clerc N., Brunser $O$.: Enteropathogens associated wjth acute diarrhea in hospitalized infants. I Pediatr Gastroenterol Nutr 1986; 5: 226-231.

18. Avánguiz P., Duffou G., Curotto D., Vergare $M$.: Síndrome diareico agudo del lactante por Rotavirus. Pedintría (Santiago) $1979 ; 22: 27-28$.

19. Avendaño L., Spencer E., Calderón A., Martinez

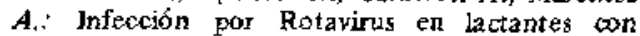
dintrea aguda. Aspectos clínicos y epidemiológioos. Rev Med Chile 1983; 111 : 240-246.

20. Avendaño L., Ojeda J., Calderón A., Mocaya J., Prenzel I., Duarte E.: Detección de Rotavirus en lactantes hospitalizados por diarrea. Rev Med Chile 1980; 108: 210-213.

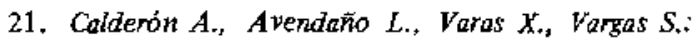
Detección de Rotavirus en lactantes con diarrea aguda, hospitalizados y ambulatorios, en Santiago de Chile, Bol Med Hosp Infant Mex 1982; 39 : 89-91.

22. Wu E., Grodo C, Avenda Ho F, Corey G.: Etjología viral de la diarrea infantì. Rev Chil Pediatr 1975; 46: 119-124.

23. Corey G.: Algunos aspectos etiológicos y epidemio- 
lógicos del síndrome diarreico agudo infantil. Rev Chil Pediatr 1975; 46: 251-263.

24. Avendaño L., Duffau G., Emilfork $M$. et al. Rotavirus en diarrea de evolución prolongada. Rev Chi Pediatr 1984; 55: 94-97.

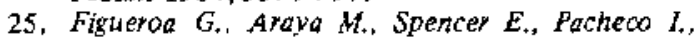
Brunser $O .:$ Diarrea aguda en lactantes menores de un año. Estudio en terreno. XXII Reunión Anual de la Sociedad Latinoamericana de Investigación Pediátrica. Los Andes, Chile, noviembre 1984.

26. Fernández $H$. et al. Aislamiento de Campylobacter jejunj en niños con diarrea aguda y cont roles sanos. Rev Med Chile 1984; 112: 238.

27. Puga $S_{\text {.: }}$ Protozoos y helmintos intestinales en lactantes de la ciudad de Valdivia, Chile. Bol Chil Parasitol 1973; $28: 39-40$.
28. Rubio M.: Encuesta enteroparasitológica en el Hospital L.C. Mackenna en Santiago. Bol Chil Parasitol 1962;17:93-100.

29. Frenzel A., Torres P., Guerrero S., Gesche IV, Monte-fusco A., Marin F.: Parasitosis intestinal en lactantes y su relación oon la infección de sus manipuladores de alimentos y el sanamiento ambiental. Rev Med Chile 1979; 107:343-351.

30. Noemi I., Reyes H., Matro G.: Infecciones por enteroparásitos en riños de sala cuna y jardín infantil de das hospitales de Santiago. Rev Chil Pediatr 1975; 46: 50-53.

31. Pérez C. Vallejos $M$.: Enteroparasitosis infantil en el Area Metropolitana de Salud Central de Santiago. Rev Chil Pediatr $1977 ; 48: 161-163$. 